\title{
The optical assembly of nanoparticles
}

\author{
David Andrews and Luciana Dávila Romero
}

Laser light can induce binding or repulsive forces between particles and thus facilitate manipulation of nanoparticle arrays.

Individual stable atoms and molecules are seldom found far apart unless they are gases. Most matter exists in a condensed phase whose cohesion and integrity are due to fundamental interactions among the particles. The balance between dispersion forces of attraction and short-range electron-cloud repulsionsas in the Lennard-Jones potential-supports the formation of stable equilibrium structures. Remarkably, off-resonant laser radiation can induce additional, optomechanical forces between particles. A publication by Michael Burns and his team ${ }^{1}$ verified this effect experimentally for a simple system of two identical, spherical particles.

The terms 'optical binding' and 'optical matter,' which have recently gained currency, imply possibilities for an interplay with other interactions, such as chemical bonding. Such forces offer distinctive features that can be exploited for controlled optical manipulation of matter. ${ }^{2}$ Progress in theory is simultaneously developing along several fronts, with many investigations using classical descriptions of the radiation field. Some studies involve the demanding experimental challenges of engaging laser light to achieve the coherent manipulation and assembly of atoms and molecules.

We are among several teams developing further applications based on the theory of quantum electrodynamics, ${ }^{3-5}$ which properly accounts for the quantized nature of the electromagnetic fields coupling the particles. With a comprehensive analysis of the potential-energy landscapes generated in a radiation field, we could potentially control the microstructure of particle assemblies. For example, some of our first calculations indicated that we could optically engineer the morphology of deposited carbon-nanotube films. ${ }^{6}$

We established that optical binding forces separate particles according to the positions of the different minima in the energyshift landscapes. ${ }^{7}$ In the typical results shown in Figure 1, local minima distinguish stable optical binding configurations. In Fig-

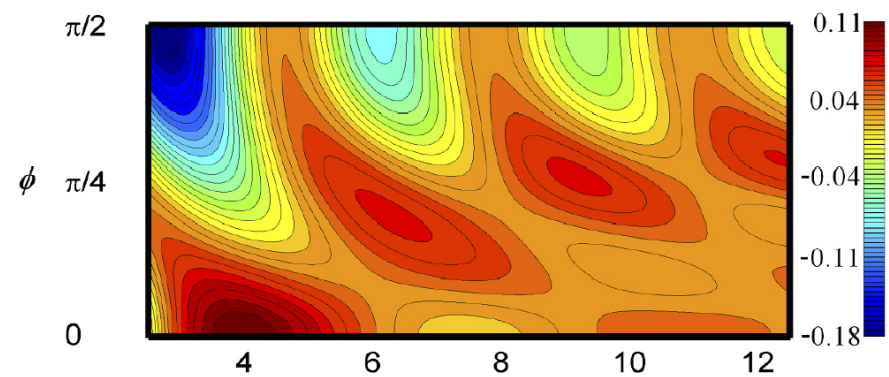

(a)
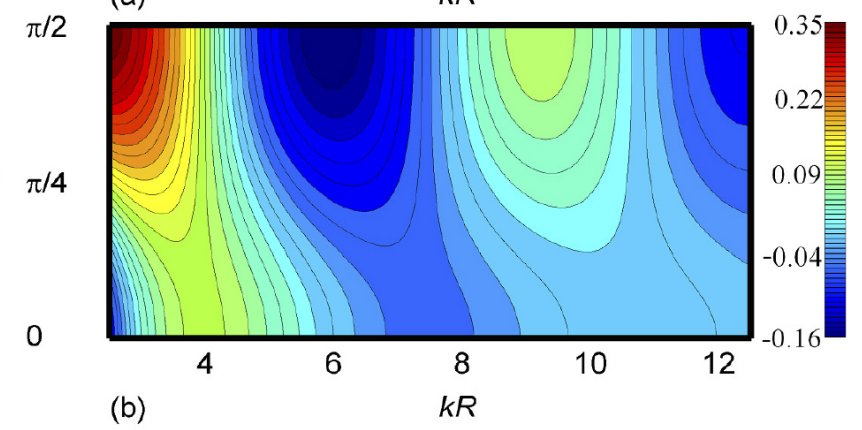

(b)

Figure 1. Landscapes of optically induced potential energy as a function of the particle separation, $R$, and the angle $\phi$ between the laser polarization and $R$ (where $k=2 \pi / \lambda$ and $\lambda$ is the wavelength): (a) $R$ coplanar with the laser propagation and polarization, (b) laser propagation perpendicular to $R$.

ure 1(a), prominent minima arise from a longitudinal configuration, where the optical beam propagates collinearly with the particles. Figure 1(b) represents a transverse configuration. For particles situated away from any local minimum, the physical significance is a force of attraction towards a neighboring point of stability. Most systems in an arbitrary configuration will be subject to both forces and torques.

Templates such as Figure 1 can be used to establish the energy landscape for a larger number of particles. Multiple occurrences of local minima and maxima enable a variety of assemblies, determined by the optically engineered conditions. The simplest

Continued on next page 

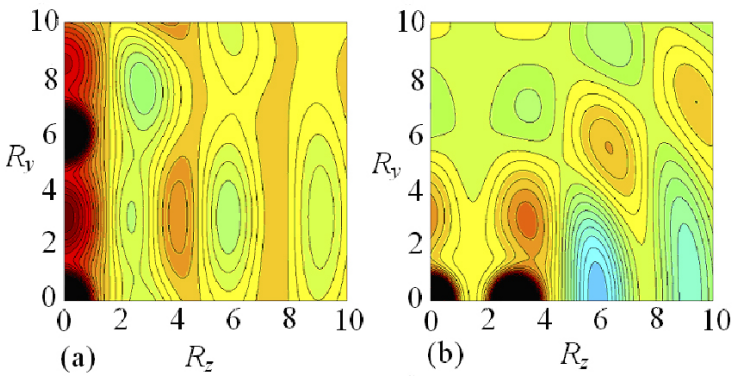

(a) $\quad R_{z}$

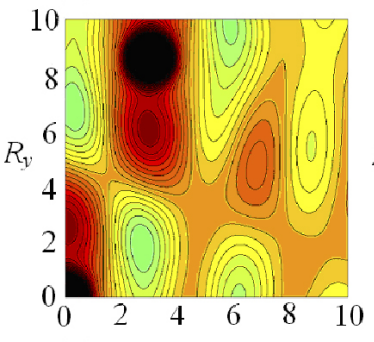

(c)

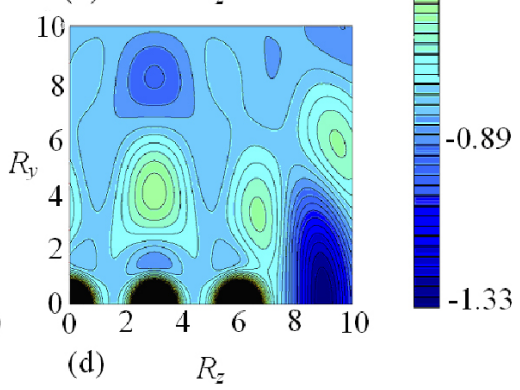

(d)

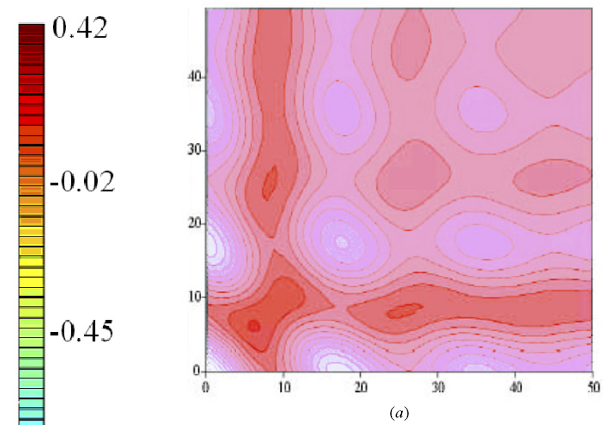

Figure 3. (a) Contour graphs of the potential energy of three particles on a ring. Ordinate and abscissa axes represent the angular displacement between each adjacent pair. (b) Clustering of nanoparticles in a twisted beam.

pair interactions, preparing for a full analysis of multiparticle assembly processes.

Work in the quantum-electrodynamics group at the University of East Anglia is supported by the UK's Engineering and Physical Sciences Research Council.

\section{Author Information}

observed assemblies are chain structures, based on a repeated superposition of the energy landscape, displaced by multiples of the closest equilibrium distance. Many more complex structures can also be formed, which we can readily identify from calculations based on three or more particles (see Figure 2).

We are specifically interested in particle behavior in twistedwavefront beams (see Figure 3). Particles trapped in the annular intensity distributions of such beams can fall into position and generate stable ring formations when counter-propagating beams are used to offset any linear propulsion due to radiation pressure. When the general analysis is extended to four or more particles, a variety of more complex 2D and 3D arrays can be produced optically. Our ongoing research focuses on the detailed properties of such assemblies, including their switchable collapse ${ }^{8}$ and the effects of tailoring the line shape of the radiation.

The development of this new area of optical binding opens up a wide range of new opportunities in the well-established field of optical trapping. Exploitation of particle properties with cleverly engineered optical configurations extends from the optically driven assembly of micro- and nanoparticles suspended in liquid media down to the manipulation of individual molecules held in cold atom traps. Already, astonishing progress has been made. At present, our main task is to extend the theory beyond

\section{David Andrews and Luciana Dávila Romero \\ University of East Anglia \\ Norwich, UK \\ http:/ / www.uea.ac.uk/ c051}

David Andrews leads research on fundamental molecular photonics and energy transport, optomechanical forces, and nonlinear optics. He has published over 200 research papers and ten books. His current research focuses on mechanisms for optical nanomanipulation and switching, and for light harvesting in nanostructured systems.

Luciana Dávila Romero is a postdoctoral research associate whose main research interests are light-matter interactions based on quantum electrodynamics. She has studied nonlinear optical processes, the angular momentum of light, molecular interactions, and optical binding. 


\section{References}

1. M. M. Burns, J. M. Fournier, and J. A. Golovchenko, Optical matter: crystallization and binding in intense optical fields, Science 249 (4970), pp. 749-754, 1990. doi:10.1126/science. 249.4970 .749

2. M. Dienerowitz, M. Mazilu, and K. Dholakia, Optical manipulation of nanoparticles: a review, J. Nanophoton. 2 (1), p. 021875, 2008. doi:10.1117/1.2992045

3. D. S. Bradshaw and D. L. Andrews, Optically induced forces and torques: interactions between nanoparticles in a laser beam, Phys. Rev. A 72 (3), p. 033816, 2005. doi:10.1103/PhysRevA.72.033816

4. A. Salam, Two alternative derivations of the static contribution to the radiationinduced intermolecular energy shift, Phys. Rev. A 76 (6), p. 063402, 2007. doi:10.1103/PhysRevA.76.063402

5. L. C. Dávila Romero and D. L. Andrews, Nanoscale optics: interparticle forces, in, D. L. Andrews ed., Structured Light and Its Applications: An Introduction to Phase-Structured Beams and Nanoscale Optical Forces, pp. 79-105, Academic Press, Burlington MA, 2008. ISBN-10: 0-12-374027-4

6. D. L. Andrews and D. S. Bradshaw, Laser-induced forces between carbon nanotubes, Opt. Lett. 30 (7), pp. 783-785, 2005. doi:10.1364/OL.30.000783

7. J. Rodríguez, L. C. Dávila Romero, and D. L. Andrews, Optical binding in nanoparticle assembly: potential energy landscapes, Phys. Rev. A 78 (4), p. 043805, 2008. doi:10.1103/PhysRevA.78.043805

8. D. L. Andrews and J. Rodríguez, Collapse of optical binding under secondary irradiation, Opt. Lett. 33 (16), pp. 1830-1832, 2008. doi:10.1364/OL.33.001830 